\title{
Mechanism of Action of Veverimer: A Novel, Orally Administered, Nonabsorbed, Counterion-Free, Hydrochloric Acid Binder under Development for the Treatment of Metabolic Acidosis in Chronic Kidney Disease
}

\author{
Gerrit Klaerner, Jun Shao, Kalpesh Biyani, Matthew Kade, Paul Kierstead, Randi Gbur, \\ Scott Tabakman, Son Nguyen, and Jerry Buysse \\ Tricida, Inc., South San Francisco, California (G.K., J.S., K.B., M.K., P.K., R.G., S.T., S.N.) and FFV Consulting, Los Altos, \\ California (J.B.)
}

Received June 30, 2020; accepted August 31, 2020

\begin{abstract}
Current management of metabolic acidosis in patients with chronic kidney disease (CKD) relies on dietary intervention to reduce daily endogenous acid production or neutralization of retained acid with oral alkali (sodium bicarbonate, sodium citrate). Veverimer is being developed as a novel oral treatment for metabolic acidosis through removal of intestinal acid, resulting in an increase in serum bicarbonate. Veverimer is a freeamine polymer that combines high capacity and selectivity to bind and remove hydrochloric acid $(\mathrm{HCl})$ from the gastrointestinal (Gl) tract. In vitro studies demonstrated that veverimer had a binding capacity of $10.7 \pm 0.4 \mathrm{mmol} \mathrm{HCl}$ per gram of polymer with significant binding capacity $(>5 \mathrm{mmol} / \mathrm{g})$ across the range of $\mathrm{pH}$ values found in the human Gl tract (1.5-7). Upon protonation, veverimer bound chloride with high specificity but showed little or no binding of phosphate, citrate, or taurocholate $(<1.5 \mathrm{mmol} /$ $\mathrm{g})$, which are all anions commonly found in the human Gl tract. Administration of veverimer to rats with adenine-induced CKD and metabolic acidosis resulted in a significant increase in fecal chloride excretion and a dose-dependent increase in serum bicarbonate to within the normal range compared with untreated
\end{abstract}

controls. Absorption, distribution, metabolism, and excretion studies in rats and dogs dosed with ${ }^{14} \mathrm{C}$-labeled veverimer showed that the polymer was not absorbed from the Gl tract and was quantitatively eliminated in the feces. Acid removal by veverimer, an orally administered, nonabsorbed polymer, may provide a potential new treatment for metabolic acidosis in patients with CKD.

\section{SIGNIFICANCE STATEMENT}

Metabolic acidosis is a complication of chronic kidney disease (CKD) as well as a cause of CKD progression. Veverimer is a high-capacity, selective, nonabsorbed, hydrochloric acidbinding polymer being developed as a treatment for metabolic acidosis. Veverimer binds and removes hydrochloric acid from the gastrointestinal tract, resulting in increased serum bicarbonate and the correction of metabolic acidosis. Veverimer is not an ion-exchange resin and does not deliver sodium or other counterions, and so it may be appropriate for patients with CKD with and without sodium-sensitive comorbidities.

\section{Introduction}

Metabolic acidosis is a common disorder in patients with non-dialysis-dependent, stage 3-5 chronic kidney disease

This work was supported in its entirety by Tricida, Inc.

The authors report the following disclosures regarding their relationship and roles at Tricida, Inc. G.K. is a full-time Tricida employee and a member of the Tricida Board of Directors and has stock and stock options in Tricida; G.K is listed on granted and pending Tricida patents. J.B. reports consulting fees and stock and stock options from Tricida during and outside this work; J.B. is listed on granted and pending Tricida patents. J.S., K.B., M.K., P.K., R.G., S.T., and S.N. are full-time Tricida employees and have stock and stock options in Tricida along with listings on granted and pending Tricida patents.

Primary laboratory of origin: Tricida, Inc. (South San Francisco, CA).

The work was partially published in abstract form at American Society of Nephrology, Kidney Week 2019 (Washington, D.C.)

https://doi.org/10.1124/jpet.120.000190.
(CKD) and is caused by the inability of the diseased kidney to quantitatively remove daily endogenous acid production (Alpern and Sakhaee, 1997; Hamm et al., 2015; Kraut and Madias, 2016). The result of this imbalance is that acid accumulates in the body and serum bicarbonate, the major extracellular acid buffer, and $\mathrm{pH}$ both fall from their normal ranges of 22-29 mEq/1 and 7.36-7.44, respectively (Wesson et al., 2020). Chronic metabolic acidosis is recognized clinically as a persistent reduction of serum bicarbonate to less than the lower limit of normal, which is generally $22 \mathrm{mEq} / \mathrm{l}$, in a patient with CKD and normal pulmonary function (Kraut and Madias, 2018; Raphael, 2018).

The human body continually generates acid through metabolism of the daily diet. Sources of acid include amino acids and nucleic acids, incomplete oxidation of carbohydrates, and

ABBREVIATIONS: CAS, Chemical Abstract Service; CKD, chronic kidney disease; CTAB, cetyltrimethylammonium bromide; GCTA, GI compartment transit assay; Gl, gastrointestinal; $\mathrm{HCl}$, hydrochloric acid; IC, ion chromatography; p/n, catalog number; SGF, simulated gastric fluid; SIB, simulated intestine inorganic buffer; SOB, simulated intestine organic and inorganic buffer; wt\%, weight percent. 
digestion of proteins from the diet (Szerlip, 2005; Scialla and Anderson, 2013; Hamm et al., 2015). The daily production of these endogenous nonvolatile acids is approximately 0.7-1 $\mathrm{mEq}$ per $\mathrm{kg}$ of body weight or $\sim 50$ to $>70 \mathrm{mEq} /$ day in healthy adults eating typical Western acid-producing diets (Alpern and Sakhaee, 1997; Scialla and Anderson, 2013; Goraya and Wesson, 2017). Dietary interventions in patients with CKD and metabolic acidosis comprising increased intake of fruits and vegetables or reduced animal protein intake have been shown to be effective in raising serum bicarbonate and slowing kidney disease progression (Goraya et al., 2014; Garneata et al., 2016). Additionally, oral alkali supplementation (sodium bicarbonate, sodium citrate) in patients with $\mathrm{CKD}$ and metabolic acidosis has also been shown to increase serum bicarbonate and slow kidney disease progression (de BritoAshurst et al., 2009; Phisitkul et al., 2010; Di Iorio et al., 2019; Dubey et al., 2020). These approaches reduce dietary acid intake or neutralize endogenous acid with oral bicarbonate but have the disadvantage of potentially increasing serum potassium (dietary approaches) or introducing significant sodium (oral alkali) in patients with CKD who are prone to hyperkalemia and sodium-sensitive comorbidities (Goraya and Wesson, 2019).

Acid binding and removal through the gastrointestinal (GI) tract using an amine-based nonabsorbed polymer, which results in an increase in serum bicarbonate, is a novel approach to treat metabolic acidosis without introducing deleterious counterions (e.g., sodium or potassium) or attempting long-term dietary changes. This approach mimics the physiologic response to acid removal observed in patients with persistent vomiting or during nasogastric suction, each of which results in an elevated serum bicarbonate level (Khanna and Kurtzman, 2006; Gennari and Weise, 2008; Valeur and Julsrud, 2013). The polymer restores the ability to excrete acid from the body, a function that is impaired during chronic kidney disease.

To achieve the desired clinical effect of increasing serum bicarbonate through acid removal in the GI tract, the aminebased nonabsorbed polymer requires specific chemical and physical properties that promote high capacity and selectivity for hydrochloric acid ( $\mathrm{HCl}$ ) binding. First, the polymer should have an amine capacity to bind at least $5 \mathrm{mEq}$ of proton per gram of polymer with a convenient daily dose of less than $10 \mathrm{~g} /$ day (Bushinsky et al., 2018). Once protonated, the acidbinding polymer should selectively bind chloride to ensure net acid removal from the GI tract (Turnberg et al., 1970). Additionally, the polymer should minimize binding and removal of other anions in the GI tract, such as fatty acids and bile acids, which represent precursors metabolized by the liver to bicarbonate (den Besten et al., 2013; Ríos-Covián et al., 2016).

In this report, we provide the initial in vitro and in vivo characterization of veverimer, a nonabsorbed HCl-binding polymer under development for the treatment of metabolic acidosis and slowing of kidney disease progression in patients with CKD (Bushinsky et al., 2018; Wesson et al., 2019a,b). Veverimer is composed of low-swelling, spherical polymeric beads that are approximately $100 \mu \mathrm{m}$ in diameter. Size exclusion built into the three-dimensional structure of the polymer enables preferential binding of chloride versus larger inorganic and organic anions, such as phosphate, citrate, fatty acids, and bile acids. Using a rat model of CKD, veverimer demonstrated a high capacity and selectivity for $\mathrm{HCl}$ binding and removal in the GI tract, leading to an increase in serum bicarbonate. Veverimer binds and removes $\mathrm{HCl}$ without exchanging the proton for a cation, thus representing a novel means of increasing acid excretion in patients with CKD and metabolic acidosis.

\section{Materials and Methods}

Chemicals and Reagents. Veverimer, which was previously designated TRC101 (Bushinsky et al., 2018), was synthesized at Tricida, Inc. (South San Francisco, CA). Veverimer is a highly crosslinked, aliphatic amine polymer with the chemical structure presented in Fig. 1; the chemical name for veverimer is poly(allylamine-co- $N, N^{\prime}$-diallyl-1,3-diaminopropane-co-1,2-diaminoethane). Veverimer was synthesized by first copolymerizing two monomers: allylamine hydrochloride [Chemical Abstract Service (CAS) Registry Number 10017-11-5] and $N, N^{\prime}$-diallyl-1,3-diaminopropane dihydrochloride (CAS Registry Number 205041-15-2), followed by crosslinking of the polymer with 1,2-dichloroethane (CAS Registry Number 107-06-2). Veverimer has an intrinsic high binding capacity and selectivity for chloride. The hydrochloric acid binding capacity derives from the high amine content of the monomer components comprising the polymer, and the extensive crosslinking in veverimer provides size-exclusion properties and selectivity for binding chloride over larger anions.

Physicochemical Characterization of Veverimer. The particle size distribution of veverimer was determined via laser light diffraction with a Malvern Mastersizer 3000 particle size analyzer (Malvern Instruments, Malvern, UK). In each measurement, a veverimer sample $(250 \pm 20 \mathrm{mg})$ was weighed into a closable 15 -ml plastic tube, and $5 \mathrm{ml}$ of methanol was added while vortexing at low speed. The tube was closed tightly, and vortexing continued at a higher speed for an additional 30 seconds. An aliquot of this suspension was then used to measure the particle size. The average for $d(0.1), d(0.5)$, and $\mathrm{d}(0.9)$ of three sample preparations as a whole number (in micrometers) was recorded.

The solubility of veverimer was evaluated in a total of 12 test media covering a range of solvent polarities as well as in test media reflecting relevant physiologic conditions veverimer encounters during transit through the GI tract (Table 1). Water, methanol, and n-heptane were evaluated to represent aqueous and organic media, respectively.

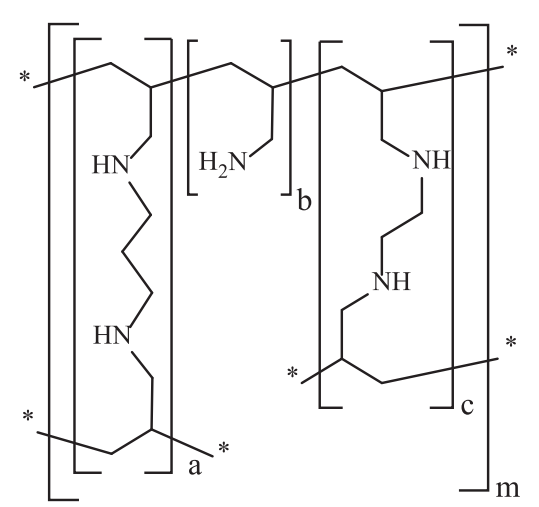

Fig. 1. Chemical structure of veverimer. Veverimer is a highly crosslinked aliphatic amine polymer that is synthesized by first copolymerizing two monomers, allylamine hydrochloride and $N, N^{\prime}$-diallyl-1,3-diaminopropane dihydrochloride, followed by crosslinking the polymer with 1,2-dichloroethane. a $=$ residue of $N, N^{\prime}$-diallyl-1,3-diaminopropane dihydrochloride (monomer and crosslinker), $\mathrm{b}=$ residue of allylamine (monomer), and $\mathrm{c}=$ residue of 1,2-dichloroethane (ethylene crosslink between two amines); an ethylene linkage between two allylamine groups is shown as an example of one of many possible linkages between amines, and $\mathrm{m}=$ a large number indicating an extended polymer network. 
TABLE 1

Solubility of veverimer in various aqueous and organic solvents

\begin{tabular}{lc}
\hline Test Medium & $\begin{array}{c}\text { Solubility } \\
(\mathrm{mg} / \mathrm{l})^{a}\end{array}$ \\
\hline Water & 0 \\
$0.1 \mathrm{~N} \mathrm{HCl}$ & 3 \\
Methanol & 0 \\
n-Heptane & 0 \\
$50 \mathrm{mM}$ acetate buffer (pH 4.5) & 0 \\
$50 \mathrm{mM}$ acetate buffer (pH 4.5) with 2 wt\% SDS & 0 \\
$50 \mathrm{mM}$ acetate buffer (pH 4.5) with 2 wt\% CTAB & 2 \\
$50 \mathrm{mM}$ acetate buffer (pH 4.5) with 2 wt\% Tween 80 & 0 \\
$50 \mathrm{mM}$ phosphate buffer (pH 6.8) & 0 \\
$50 \mathrm{mM}$ phosphate buffer (pH 6.8) with 2 wt\% SDS & 0 \\
$50 \mathrm{mM}$ phosphate buffer (pH 6.8) with 2 wt\% CTAB & 0 \\
$50 \mathrm{mM}$ phosphate buffer (pH 6.8) with 2 wt\% Tween & 0 \\
80 & \\
\hline
\end{tabular}

Tween 80 , polyoxyethylene sorbitan mono-oleate.

${ }^{a}$ Negative and zero results are reported as zero.

Water as a test medium also represented high $\mathrm{pH}$ (i.e., the equilibrium $\mathrm{pH}$ of water during the solubility test was approximately 9 ). The solution of $0.1 \mathrm{~N} \mathrm{HCl}$ and buffers at $\mathrm{pH} 4.5$ and 6.8 were used to simulate the physiologic $\mathrm{pH}$ range that exists along the GI tract. In addition, the buffers at $\mathrm{pH} 4.5$ and 6.8 were evaluated with three types of surfactants at 2 weight percent (wt\%) each: the anionic surfactant SDS (Catelog Number [p/n] 74255, CAS Registry Number 151-21-3; Sigma-Aldrich), the cationic surfactant cetyltrimethylammonium bromide (CTAB) (p/n ARK2188, CAS Registry Number 57-09-0; Sigma-Aldrich), and the nonionic surfactant polyoxyethylene sorbitan mono-oleate (Tween 80, p/n BP388, CAS Registry Number 9005-65-6; Fisher Scientific). To conduct the solubility tests, approximately $1 \mathrm{~g}$ of veverimer in 11 solvent was shaken at $37^{\circ} \mathrm{C}$ in the test medium for 7 days. The insoluble veverimer was recovered by filtering, and this was followed by washing and drying the insoluble polymer collected on the filter. The dry weight of the insoluble polymer was determined gravimetrically (ML204; Mettler Toledo) and corrected for total amount of residual counterions when applicable. The solubility was calculated as a weight loss (initial dry weight of veverimer minus dry weight of insoluble veverimer). The results were expressed as the amount of veverimer (milligram) dissolved in 11 of test medium and reported as an average value (Table 1 ).

In Vitro Hydrochloric Acid Binding Assays. The in vitro chloride binding capacity and specificity of veverimer were assessed in three biologic matrices mimicking the environment of different GI compartments: 1) simulated gastric fluid (SGF) that mimics the acidic environment of the stomach ( $\mathrm{pH} 1.2)$ and reflects an optimal condition for binding of hydrochloric acid; 2) simulated intestine inorganic buffer (SIB) with a $\mathrm{pH}$ (5.5) resembling the human proximal intestine and containing a high concentration of phosphate as a potential competing anion; and 3) simulated intestine organic and inorganic buffer (SOB) with a pH (6.2) and anion content that correspond to the human distal intestine and provide additional potential competing anions (Table 2). Veverimer was tested in duplicate at a concentration of $2.5 \mathrm{mg} / \mathrm{ml}$ at $37^{\circ} \mathrm{C}$ for 16 hours in SGF, 1 hour in SIB, or 24 hours in SOB. After incubation at $37^{\circ} \mathrm{C}$ with shaking, samples were kept at room temperature for 2 minutes without agitation, which was enough to clear the supernatant of polymer particles for sampling. Supernatant samples were then filtered and transferred to chromatography vials for ion chromatography (IC) (ICS-2100; Thermo Scientific) analysis to quantify concentrations of anions present in the supernatant before and after incubation with each of the polymers.

The chloride binding capacity of veverimer was calculated using the following equation:

Binding capacity $(\mathrm{mmol} / \mathrm{g})=\left(\mathrm{C}_{1}[\mathrm{mmol} / \mathrm{l}]-\mathrm{C}_{2}[\mathrm{mmol} / \mathrm{l}]\right) / 2.5(\mathrm{mg} / \mathrm{ml})$

Where : $C_{1}=$ chloride concentration present at the beginning $(\mathrm{mmol} / \mathrm{l})$ $\mathrm{C}_{2}=$ chloride concentration present after the incubation $(\mathrm{mmol} / \mathrm{l})$

GI Compartment Transit Assay. An in vitro GI compartment transit assay (GCTA) was used to measure the binding and retention of various anions to veverimer under conditions simulating the passage of the polymer through various compartments of the GI tract. Sequential incubation in three different matrices simulated the $\mathrm{pH}$, ionic environment, and residence times associated with transit through three different GI compartments (i.e., stomach, distal small intestine/cecum, and colon). After incubation in each the three matrices, all remaining bound ions were eluted from veverimer and quantitated by IC (ICS-2100; Thermo Scientific).

The general procedure for the GCTA assay comprised the following steps: $2.5 \mathrm{mg} / \mathrm{ml}$ of the polymer was incubated in SGF buffer at $37^{\circ} \mathrm{C}$ for 1 hour, and after incubation, the polymer was isolated and washed with $10 \mathrm{ml}$ of deionized water. The recovered polymer was next incubated in SOB buffer at $37^{\circ} \mathrm{C}$ for 2 hours; after incubation, the polymer was isolated and washed with $10 \mathrm{ml}$ of deionized water. Finally, the recovered polymer was incubated in retention buffer (50 mM N,N-bis(2-hydroxyethyl)-2-aminoethanesulfonic acid, $100 \mathrm{mM}$ acetate, $17 \mathrm{mM}$ chloride, $2.0 \mathrm{mM}$ phosphate, $3 \mathrm{mM}$ sulfate, $30 \mathrm{mM}$ bicarbonate, $\mathrm{pH} 7.0$ ) at $37^{\circ} \mathrm{C}$ for 42 hours; after incubation, the polymer was isolated and washed with $10 \mathrm{ml}$ deionized water. After this final wash, the polymer was incubated in $0.20 \mathrm{M}$ sodium hydroxide solution at $37^{\circ} \mathrm{C}$ for 18 hours to elute anions, which were quantitated by IC analysis.

pH-Dependent Equilibrium Chloride Binding. To determine the $\mathrm{pH}$-dependent equilibrium chloride binding, veverimer $(4 \mathrm{mg} / \mathrm{ml})$ was incubated for 18 hours in $100 \mathrm{mM}$ sodium chloride solution (i.e., test solution). The sample was continuously stirred during the incubation, and the $\mathrm{pH}$ of the solution was maintained at a predetermined value by slowly adding $0.1 \mathrm{~N} \mathrm{HCl}$ using an automatic titrator (T50; Mettler Toledo). The $\mathrm{pH}$ values maintained in the individual test solutions ranged from 1.5 to 7.0. After incubation, the chloride content in the supernatant was measured by IC (ICS-1600; Thermo Scientific). Equilibrium chloride binding to veverimer was calculated by determining the difference between the amount of chloride added during the experiment and the amount remaining in the supernatant at the end of the study.

Animal and Ethics Approval. All animal experiments were conducted in accordance with the Guide for the Care and Use of Laboratory Animals from the Institute for Laboratory Animal Research, National Research Council and as adopted and promulgated by US National Institutes of Health and approved by the vendor

TABLE 2

Anion concentration of in vitro test matrices designed to mimic GI tract compartments

\begin{tabular}{lcccccc}
\hline Matrix & Acetate $(\mathrm{mM})$ & Chloride $(\mathrm{mM})$ & Phosphate $(\mathrm{mM})$ & Citrate $(\mathrm{mM})$ & Taurocholate $(\mathrm{mM})$ & Oleic Acid $(\mathrm{mM})$ \\
\hline $\mathrm{SGF}^{a}$ & 0 & 98.0 & 0 & 0 & 0 & 0 \\
$\mathrm{SIB}^{b}$ & 0 & 36.0 & 20.0 & 0 & 0 & 0 \\
$\mathrm{SOB}^{c}$ & 50.0 & 36.0 & 7.0 & 1.5 & 5.0 & 30.0 \\
\hline
\end{tabular}

${ }^{a} \mathrm{SGF}, \mathrm{pH}=1.2$; it mimics the acidic environment of the fasted stomach and reflects an optimal condition for binding of $\mathrm{HCl}$.

${ }^{b} \mathrm{SIB}, \mathrm{pH}=5.5 ; \mathrm{pH}$ is representative of the human proximal small intestine (duodenum and early jejunum), and that contains chloride and a high concentration of phosphate as a potential competing anion to chloride. The solution was buffered by 2 -( $N$-morpholino)ethanesulfonic acid.

${ }^{c} \mathrm{SOB}, \mathrm{pH}=6.2 ; \mathrm{pH}$ and competing anion content (bile acid, citrate, oleic acid, and acetate) represent components of the human distal small intestine (late jejunum, ileum) and cecum. The solution was buffered by $N, N$-bis(2-hydroxyethyl)-2-aminoethanesulfonic acid. 
institutions' Animal Care and Use Committee prior to the initiation of the studies. Rat studies testing the mechanism of action and pharmacology of veverimer in the adenine-induced rat model of $\mathrm{CKD}$ and metabolic acidosis were carried out at Murigenics, Inc. (Vallejo, CA).

Male Sprague-Dawley rats ( 7 to 8 weeks, $260-280$ g) were obtained from Charles River Laboratories (Hollister, CA) for the purposes of this study and used to evaluate the pharmacodynamics of veverimer in the CKD model with respect to fecal chloride binding and effect on serum bicarbonate. The animals were pair-housed in a temperaturecontrolled facility under a 12-hour light/12-hour dark regimen. Diet and water were provided ad libitum. Adult Wistar Han rats and Beagle dogs as well as juvenile Sprague-Dawley rats were used for bioavailability assessments of $\left[{ }^{14} \mathrm{C}\right]$-veverimer absorption, distribution, metabolism, and excretion and were tested and housed at MPI/Charles River (Mattawan, MI).

Veverimer Proof-of-Concept Efficacy Study in Rats. Veverimer was evaluated in vivo in an adenine-induced rat model of CKD and metabolic acidosis (Diwan et al., 2018). The study was designed in two parts (Fig. 2). In both parts, male Sprague-Dawley rats $(N=10 /$ group) were first administered adenine $(0.75 \mathrm{wt} \%$ in casein diet [Research Diets, New Brunswick, NJ]) for 2 weeks to induce chronic kidney disease. Part 1 of the study investigated the effect of early treatment with veverimer administered to rats on a casein diet with $0.25 \mathrm{wt} \%$ adenine for the 4 weeks after the 2 -week induction period. Part 2 of the study investigated the effect of delayed treatment with veverimer; in this part, after the 2 -week induction period, rats were maintained on a casein diet containing $0.25 \mathrm{wt} \%$ adenine for 5 weeks prior to initiation of veverimer administration and through the dosing phase with veverimer. During the veverimer treatment periods, rats were fed diets admixed with $0,1.5,3.0$, and $4.5 \mathrm{wt} \%$ veverimer. After correcting for body weight and food consumption, the 1.5, 3.0, and 4.5 $\mathrm{wt} \%$ veverimer treatment groups received mean veverimer doses of $0.8,1.5$, and $2.4 \mathrm{~g} / \mathrm{kg}$ per day, respectively, in part 1 of the study and $0.7,1.4$, and $2.3 \mathrm{~g} / \mathrm{kg}$ per day, respectively, in part 2 of the study.

Study parts 1 and 2 both assessed the effect of withdrawing veverimer after the end of the treatment phase, with a 2 -week withdrawal phase in which veverimer was discontinued in the low (1.5 wt\%) and high (4.5 wt\%) veverimer dose groups but was continued in the mid-dose group $(3.0 \mathrm{wt} \%)$. All animals received a casein diet with $0.25 \mathrm{wt} \%$ adenine during the withdrawal phase.

Animals were randomized based on serum bicarbonate levels at baseline (i.e., after adenine induction of nephropathy and before initiation of the dosing period) so that mean baseline serum bicarbonate levels were comparable across all dose groups. Body weights and food consumption were determined weekly, allowing calculation of actual administered dose. For both part 1 and part 2 of the study, blood samples were obtained from the rats before initiation of veverimer dosing, then weekly during the dosing and withdrawal phases. Samples were analyzed for serum bicarbonate levels and $\mathrm{pH}$ values. In addition, a 24-hour fecal sample was obtained from each animal in the control and high-dose groups during weeks 4 and 6 of part 1 of the study and weeks 9 and 11 of part 2 of the study. Anions were extracted from lyophilized and homogenized fecal samples by incubating the samples with $\mathrm{NaOH}$ for 18 hours at $37^{\circ} \mathrm{C}$. Sample supernatants were analyzed for chloride and phosphate by IC (ICS2100; Thermo Scientific).

Bioavailability Assessment. The bioavailability of veverimer was assessed in pharmacokinetic studies conducted in Wistar Han rats and Beagle dogs by administering $\left[{ }^{14} \mathrm{C}\right]$-radiolabeled veverimer as a single oral dose to male and female animals at $0.0735 \mathrm{~g} / \mathrm{kg}$. The target radioactive dose was $100 \mu \mathrm{Ci} / \mathrm{kg}$. Absorption was characterized by measuring levels of radioactivity in the blood. The doses used in the absorption studies were selected to be representative of doses that will

Study diet

\begin{tabular}{|c|c|c|c|}
\hline Casein diet with & Casein diet with $0.25 \mathrm{wt} \%$ adenine \\
$0.75 \mathrm{wt} \%$ adenine &
\end{tabular}

Study group

1 No treatment $\quad$ No treatment

\begin{tabular}{l|l|l|l|}
\hline & \\
\hline & & \\
\hline
\end{tabular}

Study week

\begin{tabular}{|l|l|l|l|l|l|l|l|l|l|l|l|l|l|l|}
1 & 2 & 3 & 4 & 5 & 6 & 7 & 8 & 9 & 10 & 11 & 12 & 13 \\
\hline
\end{tabular}

Sample collection

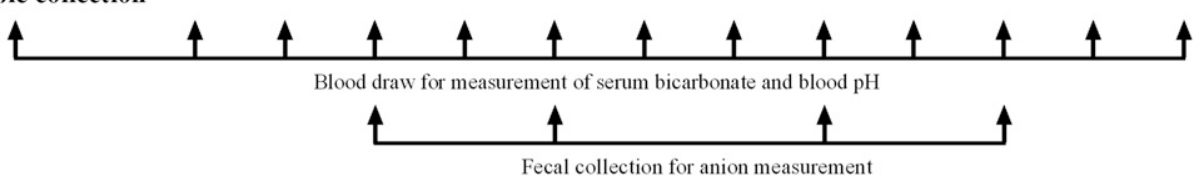

Fig. 2. In vivo evaluation of veverimer efficacy in the treatment of acidosis in an adenine-induced model of chronic kidney disease in rats. The study was designed in two parts. In both parts, male SpragueDawley rats $(N=10$ /group) were first administered adenine $(0.75 \mathrm{wt} \%$ in casein diet) for 2 weeks to induce chronic kidney disease. Part 1 of the study investigated the effect of early treatment with veverimer administered to rats on a casein diet with 0.25 wt $\%$ adenine for the 4 weeks after the 2-week chronic kidney disease induction period. In contrast, part 2 of the study investigated the effect of delayed treatment with veverimer after the 2week induction period, in which rats were maintained on a casein diet containing $0.25 \mathrm{wt} \%$ adenine for 5 weeks prior to initiation of veverimer administration and through the dosing phase with veverimer. During the veverimer treatment periods, rats were fed diets admixed with $0,1.5,3.0$, and $4.5 \mathrm{wt} \%$ veverimer. After correcting for body weight and food consumption, the $1.5,3.0$, and $4.5 \mathrm{wt} \%$ veverimer treatment groups received mean veverimer doses of $0.8,1.5$, and $2.4 \mathrm{~g} / \mathrm{kg}$ per day, respectively, in part 1 of the study and $0.7,1.4$, and $2.3 \mathrm{~g} / \mathrm{kg}$ per day, respectively, in part 2 of the study. 
be administered to human subjects, including pediatric subjects. In addition, to determine whether bioavailability differed between juvenile and adult animals because of different stages of GI maturity an oral absorption study was conducted in juvenile Sprague-Dawley rats. Rat pups on postnatal days 7 and $11(n=18 /$ sex per group) were administered $\left[{ }^{14} \mathrm{C}\right]$-radiolabeled veverimer at a total labeled/unlabeled combined veverimer dose of $0.15 \mathrm{~g} / \mathrm{kg}$ (target radioactive dose = $100 \mu \mathrm{Ci} / \mathrm{kg}$ ). Serial blood samples were taken from each animal before dosing and at $0.5,1,2,4,6,24$, and 48 hours postdose in the adult rat before dosing and at $0.5,1,2,4,6,12,24,48,72,120$, and 168 hours postdose in the dog and 1, 2, 4, 8, and 24 hours postdose in the juvenile rat. Absorption was characterized by measuring levels of radioactivity in whole blood (dog and juvenile rat) and/or plasma (dog, adult rat, and juvenile rat) using liquid scintillation counting (Packard 2300TR; PerkinElmer, Waltham, MA).

To examine distribution of veverimer, adult Wistar Han rats $(n=7 /$ sex per group) received a single oral administration of $\left[{ }^{14} \mathrm{C}\right]$-veverimer at a target dose level of $0.0735 \mathrm{~g} / \mathrm{kg}(100 \mu \mathrm{Ci} / \mathrm{kg})$. At each time point evaluated $(0.5,1,6,24,48,120$, and 168 hours postdose), rats $(n=1 /$ sex per time point) were euthanized, and carcasses were subjected to quantitative whole-body autoradiography using a Fuji FLA-5100 fluorescent image analyzer (Fuji Photo Film Co., Ltd., Japan).

To determine excretion of veverimer, adult Wistar Han rats $(n=3$ / sex) and adult Beagle dogs $(n=3 /$ sex $)$ received a single oral administration (via gavage) of $\left[{ }^{14} \mathrm{C}\right]$-veverimer at $0.0735 \mathrm{~g} / \mathrm{kg}(100 \mu \mathrm{Ci} / \mathrm{kg})$. Animals were placed in metabolic cages immediately after dosing, and urine, feces, expired air (rat only), and cage washings were collected predose and at the following postdose time intervals:

Urine (Rat and Dog): 0-6 (rat) or 0-12 (dog), 6-24 (rat) or 12-24 (dog), 24-48, 48-72, 72-96, 96-120, 120-144 and 144-168 hours.

Feces (rat and dog): 0-24, 24-48, 48-72, 72-96, 96-120, 120-144, and 144-168 hours.

Expired air (rat): 0-24, 24-48, and 48-72 hours.

Cage washings (dog): 24, 48, 72, 96, 120, 144, and 168 hours.

Cage washings (rat): 168 hours.

Rats were euthanized after the 168-hour sample collection, and additional samples for radioactivity measurements were taken from carcasses. All rat and dog samples were analyzed using liquid scintillation counting (Packard 2300TR; PerkinElmer).

\section{Results}

Veverimer Particle Size Distribution and Solubility in Aqueous and Organic Solvents. The physical form of veverimer is that of a free-flowing powder composed of lowswelling, spherical beads (Fig. 3). Each bead is a single, high-molecular weight, crosslinked polyamine molecule. The particle size of veverimer was primarily controlled by the stir rate during the suspension polymerization reaction in which the beads are first formed to generate an average particle diameter of approximately $100 \mu \mathrm{m}$. Particle size distribution analysis showed a monomodal particle size distribution, wherein greater than $90 \%$ of the volume was composed of particles with a diameter larger than $40 \mu \mathrm{m}$ (Fig. 3).

The solubility of veverimer was evaluated in a variety of aqueous and organic solvents and expressed as the amount of veverimer (milligram) dissolved in $1 \mathrm{l}$ of solvent (Table 1). Apart from the $0.1 \mathrm{~N} \mathrm{HCl}$ (veverimer solubility of $3 \mathrm{mg} / \mathrm{l}$ ) and $50 \mathrm{mM}$ acetate buffer (pH 4.5) with $2 \mathrm{wt} \%$ CTAB (veverimer solubility of $2 \mathrm{mg} / \mathrm{l}$ ) matrices, veverimer was uniformly insoluble in the aqueous and organic solvents tested. In accordance with USP (General Notices and Requirements), the descriptive term of insoluble or practically insoluble is applied to any material wherein 10,000 or more parts of solvent are required for one part of solute, and thus veverimer is determined to be insoluble in aqueous and organic solvents and in media encompassing the physiologic $\mathrm{pH}$ range with and without surfactant.

Characterization of Veverimer Hydrochloric Acid Binding In Vitro at Physiologically Relevant pH Values. Three in vitro assessments of the chloride binding capacity of veverimer were conducted in test matrices that mimicked various GI compartments with respect to $\mathrm{pH}$ and anion composition (Table 2). Competing anions (e.g., phosphate, citrate, and taurocholate) were included in the two matrices simulating intestinal fluid (SIB and SOB). Table 3 summarizes the matrices used, the incubation times, the competing anions, and the binding of chloride and competing anions by veverimer. Binding studies in the test matrices demonstrated that veverimer bound chloride with high capacity and selectivity. Veverimer bound $10.7 \pm 0.4 \mathrm{mmol}$ of chloride per gram of polymer in SGF. In the SIB matrix, veverimer preferentially bound chloride over phosphate with binding capacities of $4.3 \pm 0.1$ mmol of chloride and $1.5 \pm 0.3 \mathrm{mmol}$ of phosphate per gram of polymer, respectively. In the most complex SOB matrix, veverimer preferentially bound chloride $(3.8 \pm 0.3 \mathrm{mmol} / \mathrm{g}$ of polymer) in the presence of various competing inorganic and organic anions such that the polymer essentially did not bind phosphate, citrate or taurocholate.

An in vitro GCTA was used to measure the binding and retention of various anions to veverimer under conditions simulating the passage of the polymer through distinct compartments of the GI tract. Sequential incubation in three different matrices simulated the $\mathrm{pH}$, ionic environment and residence times associated with transit through the stomach (SGF buffer), distal small intestine/cecum (SOB buffer), and colon (retention buffer). Veverimer bound an average of 8.1 mmol of chloride per gram of polymer under simulated gastric conditions $\left(\mathrm{pH} 1.2,1\right.$ hour, $\left.37^{\circ} \mathrm{C}\right)$ and retained most of the bound chloride $(7 \mathrm{mmol} / \mathrm{g}$ of polymer; approximately $85 \%)$ after being challenged by conditions designed to mimic the environment of the distal small intestine and cecum $(\mathrm{pH} \mathrm{6.2,}$ 2 hours, $37^{\circ} \mathrm{C}$ ). After the third incubation under conditions representing the colon ( $\mathrm{pH} 7.0,42$ hours, $37^{\circ} \mathrm{C}$ ), approximately $60 \%$ of initially bound chloride (approximately $5 \mathrm{mmol}$ chloride/per gram of polymer) was retained. Release of chloride was attributed to the $\mathrm{pH}$-dependent decrease in the total binding capacity of the veverimer polymer. Veverimer bound very little or no measurable amounts of the other competing anions (i.e., acetate, phosphate, citrate, taurocholate, oleic acid, sulfate, and bicarbonate) present in the simulated human distal small intestine/cecum and colon matrices.

$\mathrm{pH}$-dependent equilibrium chloride binding of veverimer $(4 \mathrm{mg} / \mathrm{ml})$ was determined in $100 \mathrm{mM}$ sodium chloride solutions (i.e., test solution) in which the $\mathrm{pH}$ of the solution was maintained at a predetermined value by slowly adding $0.1 \mathrm{~N} \mathrm{HCl}$. The $\mathrm{pH}$ values maintained in the individual test solutions ranged from 1.5 to 7.0. Although the chloride binding capacity for veverimer decreased with increasing $\mathrm{pH}$, which was considered related to a decrease in veverimer protonation, veverimer maintained significant chloride binding capacity (i.e., $>5 \mathrm{mmol} / \mathrm{g}$ of polymer) over a range of $\mathrm{pH}$ values (1.5-7.0) that are physiologically relevant for the GI tract (Fig. 4).

Veverimer Effects on Serum Bicarbonate and Fecal Chloride Excretion in Rats with CKD and Metabolic Acidosis. Veverimer was evaluated in a two-part in vivo study in male Sprague-Dawley rats ( $n=10$ /group) administered 

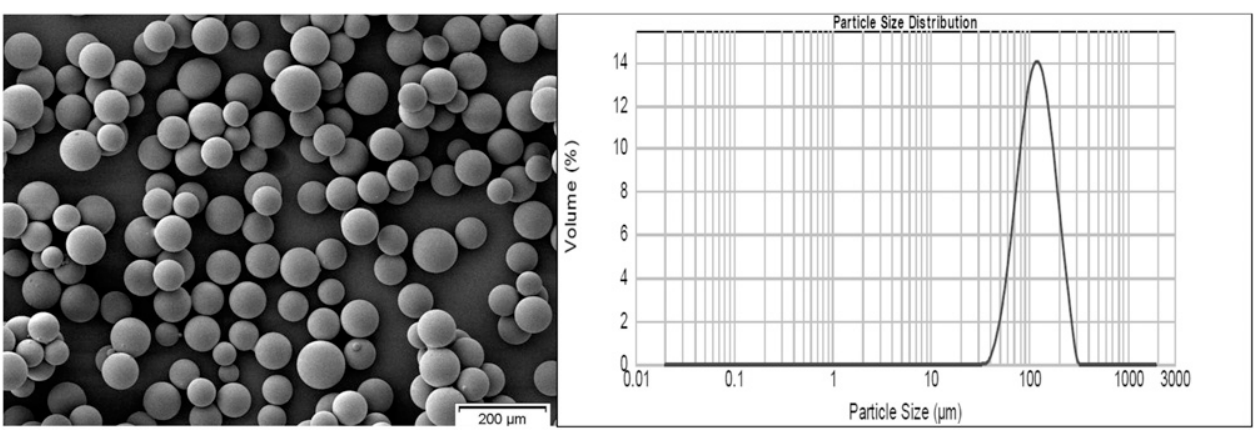

Fig. 3. Veverimer beads and monomodal particle size distribution. The particle size of veverimer was controlled in the first step of the drug substance synthetic process. Veverimer polymer beads were synthesized by a polymerization process that maintained monomer droplets in the suspension medium before initiation of the polymerization step. The suspended monomer droplets were converted to crosslinked polymer particles that allowed for control of particle size. Veverimer beads were characterized using laser diffraction, and a monomodal distribution was detected, wherein greater than $90 \%$ of the volume was composed of beads with a particle size diameter larger than $40 \mu \mathrm{m}$.

adenine ( $0.75 \mathrm{wt} \%$ in casein diet) for 2 weeks to induce CKD (Fig. 2). The veverimer-related effects on serum bicarbonate and fecal excretion of chloride and phosphate are summarized in Figs. 5 and 6. Animals were randomized based on serum bicarbonate levels at baseline (i.e., after adenine induction of CKD and before initiation of the dosing period) so that mean baseline serum bicarbonate levels were similar across all dose groups. Endpoints evaluated in the study included serum bicarbonate and $\mathrm{pH}$ (weekly measurements) and fecal chloride and phosphate (24-hour samples measured at weeks 4 and 6 of part 1 and weeks 9 and 11 of part 2). The part 1 and part 2 rat studies were designed to accommodate a withdrawal period, with one group ( $3.0 \mathrm{wt} \%$ ) maintained on continuous dosing as an internal control for the withdrawal groups tested (1.5 and $4.5 \mathrm{wt} \%)$.

Serum bicarbonate was elevated to within the normal range (23.0-31.1 $\mathrm{mEq} / \mathrm{l}$ based on values for five age-matched rats) in veverimer-dosed rats but remained below normal in untreated CKD control rats. Serum bicarbonate levels generally remained within the normal range with continued veverimer dosing, whereas withdrawal of veverimer was associated with a decline in serum bicarbonate within 2 weeks to values that were generally less than those observed in control animals. The effect of veverimer on blood $\mathrm{pH}$ reflected the results observed for serum bicarbonate (unpublished data). A significant increase in fecal chloride was observed in veverimer-dosed rats relative to untreated controls. Although veverimer also significantly increased fecal phosphate excretion, the effect was notably less than that observed for chloride. The study results support the proposed veverimer mechanism of action (i.e., selective binding, retention and removal of $\mathrm{HCl}$ from the body through the feces), and the CKD model provides proof-of-concept that veverimer administration results in restoration of serum bicarbonate to the normal range in a dose-dependent manner.

Veverimer Absorption, Distribution, Metabolism, and Excretion. The physicochemical properties of veverimer, including its insolubility in aqueous and organic solvents and particle size distribution, suggested that the polymer would not be absorbed from the GI tract. To test this hypothesis, the absorption, distribution, metabolism, and excretion of an administered pharmaceutical compound within an organism of $\left[{ }^{14} \mathrm{C}\right]$-veverimer after oral administration of a single dose in both Wistar Han rats and Beagle dogs was assessed.

Absorption of $\left[{ }^{14} \mathrm{C}\right]$-veverimer into the blood was not detectable in any adult rat plasma, dog blood, or juvenile rat blood or plasma sample. Radioactivity was detected in only one dog plasma sample at a single time point but was not considered to indicate absorption since the detectable level was close to the limit of detection, fell within the range of background sample radioactivity values, and was not observed in this dog at time points bracketing the sample. The results of the radiolabeled absorption studies indicated that veverimer was not systemically absorbed from the GI tract after oral administration to the juvenile or adult rat or the dog.

Distribution of veverimer was assessed by quantitative whole-body autoradiography in the adult rat. Radioactivity was not detectable in any tissue or organ other than within the GI tract, and therefore, tissue distribution could not be quantitated. The intestinal transit of the radiolabel was effectively complete by approximately 120 hours after

TABLE 3

Chloride binding by veverimer in matrices mimicking the GI tract

\begin{tabular}{lccccc}
\hline \multirow{2}{*}{ Matrix } & \multirow{2}{*}{ Incubation Time (h) } & \multicolumn{3}{c}{ Anion Bound (mmol/g Polymer) } \\
\cline { 3 - 5 } & & Chloride & Phosphate & Citrate & Taurocholate \\
\hline $\mathrm{SGF}^{a}$ & 16 & $10.7 \pm 0.4$ & N/A & N/A & N/A \\
$\mathrm{SIB}^{b}$ & 1 & $4.3 \pm 0.1$ & $1.5 \pm 0.3$ & N/A & N/A \\
SOB $^{c}$ & 24 & $3.8 \pm 0.3$ & $0.1 \pm 0.1^{d}$ & $<0.1^{e}$ & $<0.1^{e}$ \\
\hline
\end{tabular}

N/A, not applicable

${ }^{a} \mathrm{SGF}, \mathrm{pH}=1.2$; it mimics the acidic environment of the fasted stomach and reflects an optimal condition for binding of $\mathrm{HCl}$.

${ }^{b} \mathrm{SIB}, \mathrm{pH}=5.5 ; \mathrm{pH}$ is representative of the human proximal small intestine (duodenum and early jejunum), and that contains chloride and a high conc. of phosphate as a potential competing anion to chloride. The solution was buffered by 2 -( $N$-morpholino)ethanesulfonic acid

${ }^{c} \mathrm{SOB}, \mathrm{pH}=6.2 ; \mathrm{pH}$ and competing anion content (bile acid, phosphate, citrate, oleic acid, and acetate) represent components of the human distal small intestine (late jejunum, ileum) and cecum. The solution was buffered by $N, N$-bis(2-hydroxyethyl)-2-aminoethanesulfonic acid.

${ }^{d}$ Phosphate conc. used to calculate phosphate binding capacity of veverimer lots were estimated by calculating the change in the phosphate peak area before and after incubating the polymer in SOB.

${ }^{e}$ Citrate and taurocholate peaks for veverimer lots were not well separated in the IC chromatogram because of method limitations. Combined peak area was used to estimate conc. of these anions before and after incubating the polymer in SOB. Oleic acid was not determined because it was not detected by the IC method used. 


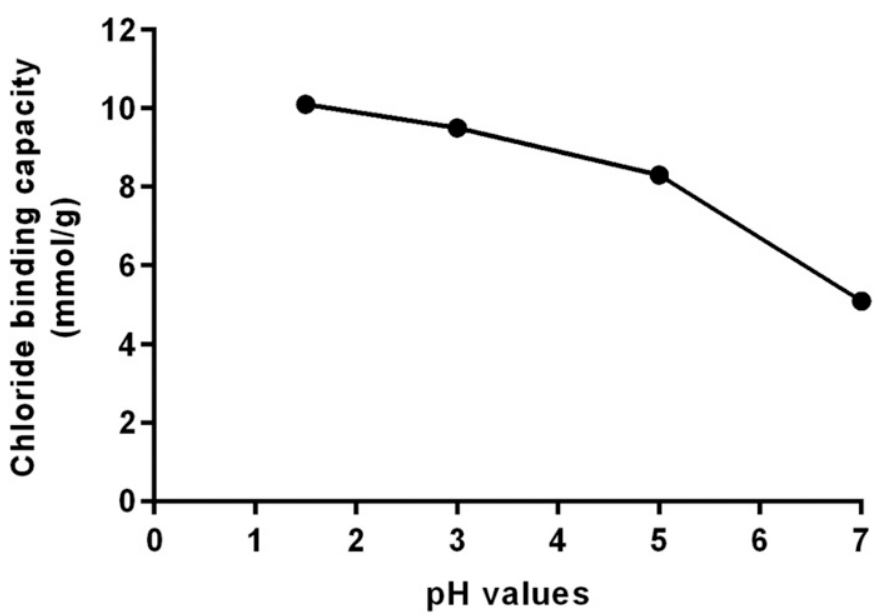

Fig. 4. Veverimer chloride binding through a range of $\mathrm{pH}$ values. Equilibrium chloride binding was assessed at different $\mathrm{pH}$ values for veverimer as described. Veverimer had a chloride binding capacity of $10.1 \mathrm{mmol} / \mathrm{g}$ under highly acidic conditions (i.e., $\mathrm{pH} 1.5$ ) in which all the available free amine groups (primary, secondary, and tertiary) were protonated and bound to chloride as a counterion. As the $\mathrm{pH}$ of the incubation solution was increased, the equilibrium chloride binding capacity of veverimer decreased but still bound $>5.0 \mathrm{mmol} / \mathrm{g}$ of chloride under physiologically relevant conditions (i.e., $\mathrm{pH}$ 5.0-7.0).

administration of $\left[{ }^{14} \mathrm{C}\right]$-veverimer. The lack of radioactivity in tissues or organs outside of the GI tract demonstrated that radiolabeled veverimer was not absorbed from the gut after oral administration to rats.
Excretion of veverimer was predominantly through the feces (Tables 4 and 5). Approximately 95\%-97\% of the administered radioactive dose in the rat and 96\%-99\% in the dog was recovered in the feces. Excretion of radioactivity was relatively rapid with most of the radioactivity (approximately 90\%) excreted within the first 24 hours postdose in both species. In general, radioactivity was not detectable in any fecal or urine sample after 72 hours postdose in the rat or after 120 hours in the dog. Very small amounts of radioactivity were detected over the first 24-hour postdose period in rat urine and expired air (mean $\leq 0.02 \%$ of the administered dose, each matrix) and dog urine (mean $=0.02 \%$ of the administered dose). The amount in urine and/or expired air for both the rat and dog corresponded within the variability of the study to the unincorporated radiolabel remaining in the administered material $\left[{ }^{14} \mathrm{C}\right]$-veverimer. In addition, some degree of crosscontamination of urine by feces was likely to have contributed to the very low levels of radiolabel detected in the urine of both species because complete separation of urine and feces is rarely completely achieved despite the use of metabolic cages. Thus, excretion data together with blood and plasma data demonstrated that veverimer was not systemically absorbed in rats and dogs after oral administration, with almost $100 \%$ of the recovered radioactivity in the feces.

Evaluation of veverimer metabolism in the rat and dog was not conducted based on the absence of systemic exposure. The in vivo pharmacology studies (summarized above) demonstrating binding and removal of chloride after oral administration of veverimer along with the significant 10 -fold increase

A

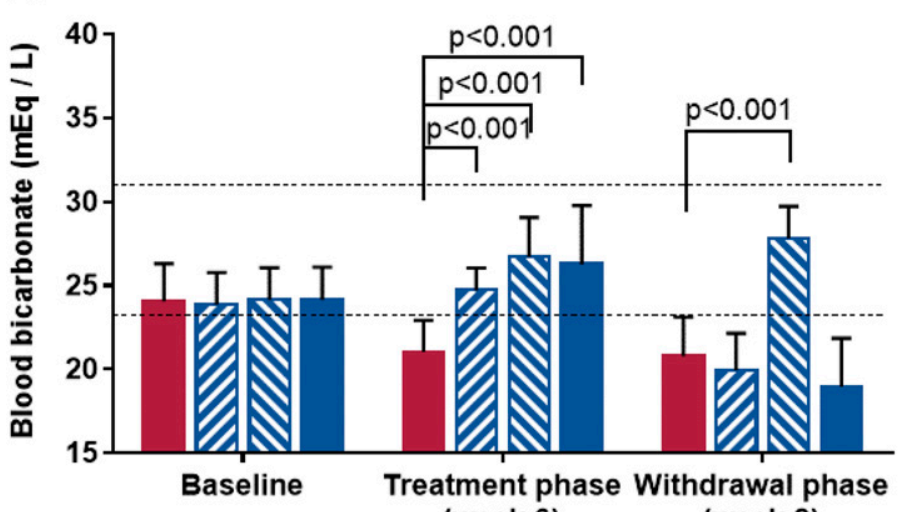

(week 6)

B

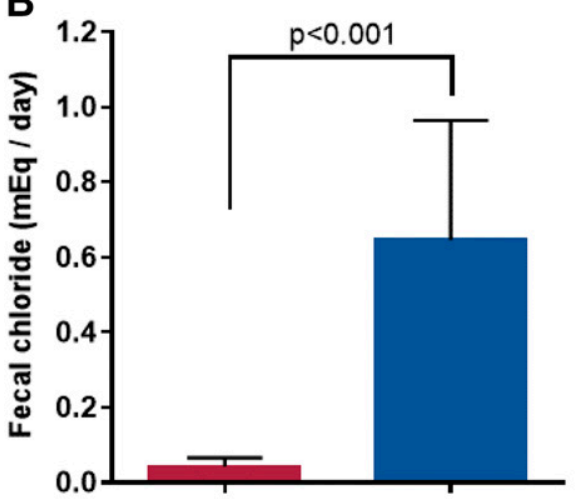

Untreated 4.5 wt $\%$ veverimer (week 8)

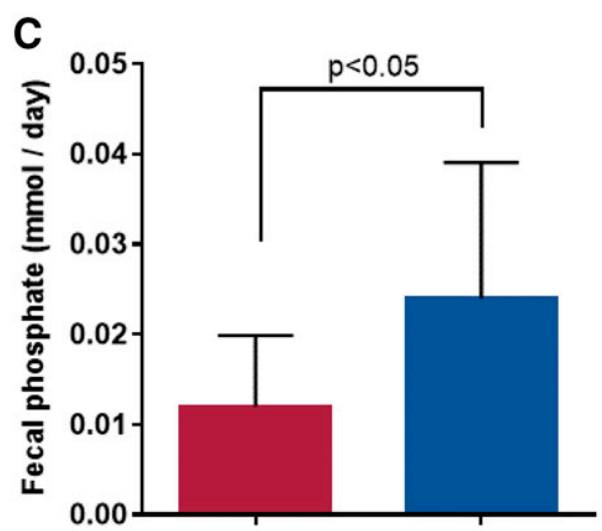

Untreated $4.5 \mathrm{wt} \%$ veverimer
Fig. 5. Effect of veverimer on serum bicarbonate and fecal excretion of chloride and phosphate in part 1 of proof-ofconcept rat study. (A) Effect of veverimer on serum bicarbonate in part 1 . Mean \pm S.D. from blood samples collected at week 6 (treatment phase) and week 8 (withdrawal phase). The $3.0 \mathrm{wt} \%$ veverimer treatment group remained on veverimer, whereas 1.5 and $4.5 \mathrm{wt} \%$ veverimer treatment groups were withdrawn from drug during the withdrawal phase (weeks 7 to 8). Horizontal dotted lines mark the normal serum bicarbonate range for male Sprague-Dawley rats of the same age. Statistical analysis: two-way ANOVA with Dunnett's multiple comparisons test vs. untreated group; $N=10$ rats per group. (B) Fecal chloride excretion in rats during part 1 of the study. Mean \pm S.D. from fecal samples collected at week 4 and week 6. Statistical analysis: unpaired, two-tailed $t$ test; $N=10$ rats per group. (C) Fecal phosphate excretion in rats during part 1 of the study. Mean \pm S.D. from fecal samples collected at week 4 and week 6. Statistical analysis: unpaired, two-tailed $t$ test; $N=10$ rats per group. 
A

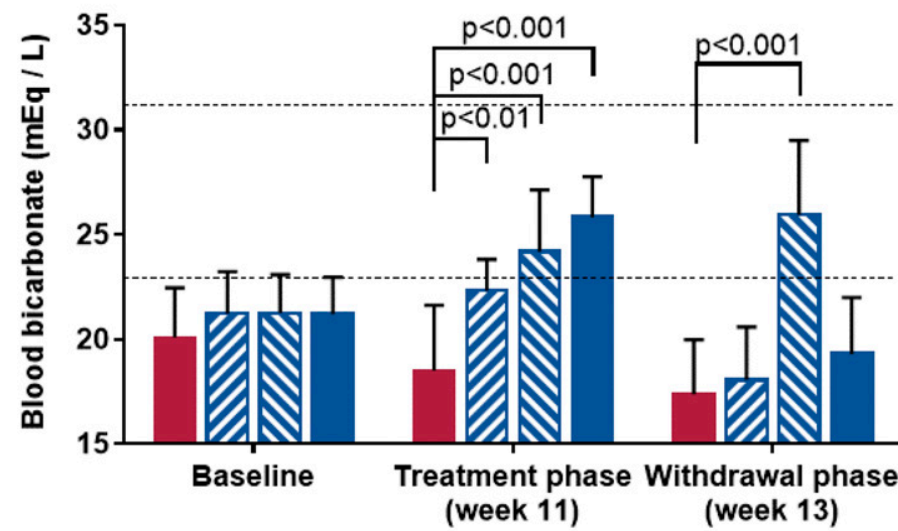

B

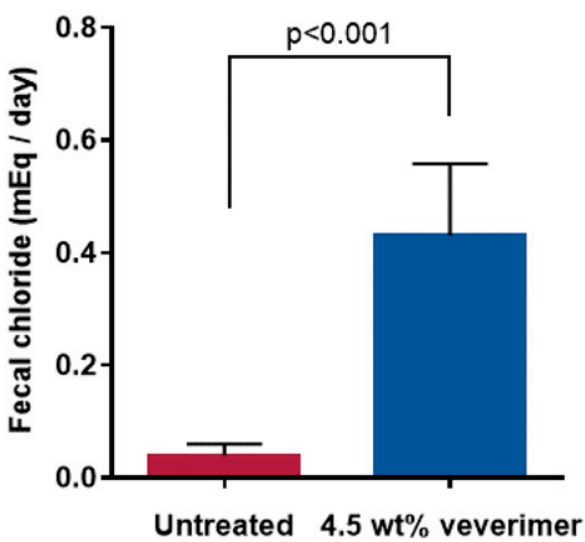

C

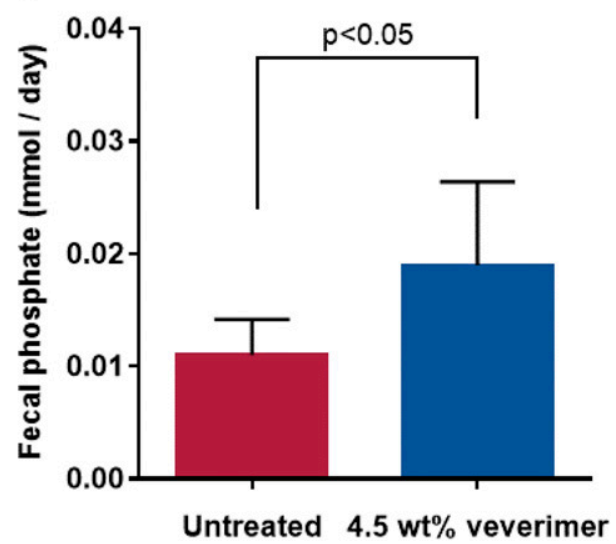

Fig. 6. Effect of veverimer on serum bicarbonate and fecal excretion of chloride and phosphate in part 2 of proof-ofconcept rat study. (A) Effect of veverimer in part 2 of the study. Mean \pm S.D. from blood samples collected at week 11 (treatment phase) and week 13 (withdrawal phase). The $3.0 \mathrm{wt} \%$ veverimer treatment group remained on veverimer, whereas 1.5 and $4.5 \mathrm{wt} \%$ veverimer treatment groups were withdrawn from drug during the withdrawal phase (weeks 12 to 13 ). Horizontal dotted lines mark the normal serum bicarbonate range for male SpragueDawley rats of the same age. Statistical analysis: two-way ANOVA with Dunnett's multiple comparisons test vs. untreated group; $N=10$ rats per group. (B) Fecal chloride excretion during part 2 of the study. Mean \pm S.D. from fecal samples collected at week 9 and week 11. Statistical analysis: unpaired, twotailed $t$ test; $N=10$ rats per group. (C) Fecal phosphate excretion during part 2 of the study. Mean \pm S.D. from fecal samples collected at week 9 and week 11. Statistical analysis: unpaired, twotailed $t$ test; $N=10$ rats per group. in fecal chloride but only a 2 -fold increase in phosphate fecal excretion relative to controls in rats with adenine-induced nephropathy administered veverimer suggested that the polymer retained its functional integrity (i.e., high capacity, selective chloride binding) and, therefore, structural integrity during transit through the GI tract after oral administration, supporting a lack of veverimer metabolism or degradation within the GI tract.

Treatment with veverimer did not cause diarrhea in the rats or dogs used in the absorption, distribution, metabolism, and excretion of an administered pharmaceutical compound within an organism assessment or in the rat model of metabolic acidosis associated with CKD. In addition, analysis of serum data for sodium, potassium, and calcium showed no change of these serum cations during the animal studies. Serum magnesium was not measured during the animal studies.

\section{Discussion}

Veverimer is an orally administered, nonabsorbed, counterionfree $\mathrm{HCl}$ binder under development for the treatment of metabolic acidosis in patients with CKD. The mechanism of action of veverimer relies on highly specific binding and retention of proton and chloride as the polymer traverses the GI tract (Fig. 7). In the first step of this process, veverimer becomes protonated after ingestion, with high-capacity proton binding occurring across the $\mathrm{pH}$ range $(\mathrm{pH}=1.5-7.0)$ found in

TABLE 4

Recovery of administered $\left[{ }^{14} \mathrm{C}\right]$-veverimer in excreta and carcasses of male and female Wistar Han rats

\begin{tabular}{|c|c|c|c|c|}
\hline \multirow{3}{*}{ Sample } & \multicolumn{4}{|c|}{$\%$ of Administered Dose } \\
\hline & \multicolumn{2}{|c|}{ Males $(n=3)$} & \multicolumn{2}{|c|}{ Females $(n=3)$} \\
\hline & Mean & S.D. & Mean & S.D. \\
\hline Total feces & 97.09 & 1.72 & 95.05 & 4.38 \\
\hline Total urine & 0.02 & 0.01 & 0.02 & 0.01 \\
\hline Total expired air ${ }^{a}$ & 0.02 & 0.01 & 0.01 & 0.01 \\
\hline Cage wash ${ }^{b}$ & ND & $\mathrm{NC}$ & ND & $\mathrm{NC}$ \\
\hline Carcass $^{b}$ & ND & $\mathrm{NC}$ & $\mathrm{ND}$ & $\mathrm{NC}$ \\
\hline Total recovery & 97.13 & 1.72 & 95.08 & 4.38 \\
\hline
\end{tabular}

$\mathrm{NC}$, not calculable; ND, radioactivity not detectable.

${ }^{a} \mathrm{Up}$ to $72 \mathrm{~h}$ postdose, as this time sampling was discontinued as per plan because of radioactivity not being detectable in the $24-48$ and $48-72$-h collections.

${ }^{b}$ At $168 \mathrm{~h}$ postdose only. 
TABLE 5

Recovery of administered $\left[{ }^{14} \mathrm{C}\right]$-veverimer in excreta and carcasses of male and female Beagle dogs

\begin{tabular}{|c|c|c|c|c|}
\hline \multirow{3}{*}{ Sample } & \multicolumn{4}{|c|}{$\%$ of Administered Dose } \\
\hline & \multicolumn{2}{|c|}{ Males $(n=3)$} & \multicolumn{2}{|c|}{ Females $(n=3)$} \\
\hline & Mean & S.D. & Mean & S.D. \\
\hline Total feces & 98.98 & 3.35 & 96.72 & 7.28 \\
\hline Total urine & 0.02 & 0.01 & 0.02 & $<0.01$ \\
\hline Total cage wash & 0.01 & 0.01 & 0.01 & 0.01 \\
\hline Total recovery & 99.01 & 3.36 & 96.74 & 7.28 \\
\hline
\end{tabular}

the GI lumen (Fig. 4; Table 3). The concurrent binding and retention of chloride by the protonated polymer facilitated by the highly crosslinked polymer backbone that restricts the binding of larger anions effectively removes $\mathrm{HCl}$ from the GI tract, which is then eliminated with the polymer in the feces (Figs. 5 and 6). $\mathrm{HCl}$ removal in this manner results in an elevation of serum bicarbonate (Figs. 5 and 6). In patients with CKD and metabolic acidosis, in which kidney-mediated acid excretion is reduced resulting in acid retention (Wesson et al., 2020), veverimer can restore an important acid excretion capacity and so is distinguished from other metabolic acidosis interventions that either reduce acid intake or neutralize systemic acidity.

The physicochemical analysis of veverimer along with the nonclinical pharmacology studies reported here illustrate various aspects of the proposed mechanism of action for veverimer. Veverimer is a free-flowing powder composed of highly crosslinked, low-swelling, spherical polymeric beads (Fig. 3). Particle size analysis showed a monomodal particle size distribution, wherein greater than $90 \%$ of the volume was composed of particles with a diameter $>40 \mu \mathrm{m}$ and an average diameter of approximately $100 \mu \mathrm{m}$. These size parameters were carefully selected to prevent systemic absorption from the GI tract while being small enough to optimize oral administration and tolerability (Bushinsky et al., 2018). The particle size distribution of the polymer beads is primarily controlled by the stir rate during the suspension polymerization in which the beads are first formed. Solubility tests in a variety of organic and aqueous solvents showed no significant solubility of the polymer. In vitro measurement of chloride binding in a range of intestinal mimics and $\mathrm{pHs}$ demonstrated that the extensive crosslinking promoted high capacity and selective $\mathrm{HCl}$ binding, particularly in the most complex matrix mimicking the lower GI tract, in which veverimer preferentially bound chloride $(3.8 \mathrm{mmol} / \mathrm{g}$ polymer $)$ in the presence of various competing anions, some of which provide precursors for bicarbonate production (Table 3). Furthermore, the retention of chloride, which was evaluated with GCTA that simulated the passage of the polymer through various compartments of the GI tract, showed approximately $60 \%$ of initially bound chloride (approximately $5 \mathrm{mmol} / \mathrm{g}$ polymer) was retained. The highly crosslinked structure of veverimer confers a marked size-exclusion selectivity to the negatively charged moieties that bind to the protonated polymer, strongly favoring binding of the smallest anions and restricting binding of larger anions. Based on the physicochemical characteristics of veverimer and the results from in vitro and human studies, veverimer is unlikely to have significant drug-drug interactions (Shao et al., 2020).
Proof-of-concept data for veverimer were provided by a rat model of adenine-induced CKD (Diwan et al., 2018) that is characterized by low serum bicarbonate $(<23.0 \mathrm{mEq} / \mathrm{l})$ and acidemia $(\mathrm{pH}<7.4)$. Dosing with veverimer initiated immediately after the 2-week adenine CKD-induction period or 7 weeks after initiation of adenine exposure at doses of $0.7-2.4 \mathrm{~g} / \mathrm{kg}$ per day by dietary admixture resulted in a significant, dose-dependent increase in serum bicarbonate within 1 week, a dose-dependent increase in blood $\mathrm{pH}$, and a significant, concomitant increase in fecal chloride excretion relative to untreated controls (Figs. 5 and 6). The pharmacological activity of veverimer-specifically maintenance of serum bicarbonate levels within the normal range-was shown to be durable through at least 6 weeks of dosing. Discontinuation of dosing was associated with a decrease in serum bicarbonate and $\mathrm{pH}$ values that were below the normal range for agematched rats, confirming the treatment effect of veverimer.

The results from the rat model of CKD are consistent with clinical findings in patients with CKD and metabolic acidosis treated with veverimer (Bushinsky et al., 2018; Wesson et al., 2019a,b). The veverimer doses at which an effect on serum bicarbonate was observed in both phase 3 clinical trials $(0.05-0.15 \mathrm{~g} / \mathrm{kg}$ per day) were lower than the doses used in the nonclinical study (0.7-2.4 $\mathrm{g} / \mathrm{kg}$ per day) reported here, but rats produce approximately 7.5 -fold more acid per $\mathrm{kg}$ of body weight than humans (Toto and Alpern, 1996; Lin et al., 1998). Correcting the rat dose by this factor (7.5) results in a dose (0.1-0.32 $\mathrm{g} / \mathrm{kg}$ per day) that is consistent with the pharmacologically active dose in humans. In addition, it is noted that the limited phosphate binding detected in the in vitro matrix experiments (Table 3 ) and the in vivo treatment experiments (Figs. 5C and 6C) did not translate into physiologic changes in serum or urinary phosphate in either animal studies or human clinical studies (Bushinsky et al., 2018; Wesson et al., 2019a,b).

Lack of veverimer absorption from the GI tract was demonstrated in both rats and dogs administered a single oral dose of $\left[{ }^{14} \mathrm{C}\right]$-veverimer. The mean bead size of approximately $100 \mu \mathrm{m}$ with a narrow particle size distribution likely restricted absorption of particles from the GI tracts of the test animals, which was consistent with literature reports showing that particles larger than $0.5 \mu \mathrm{m}$ are not systemically absorbed (Jung et al., 2000). Because radioactivity was not observed in the plasma of either species, metabolism was not evaluated. The lack of absorption in conjunction with the pharmacological effects on serum bicarbonate and fecal chloride excretion in rats supports that veverimer is not metabolized or degraded but maintains functional and, therefore, structural integrity during transit through the GI tract after oral administration.

Veverimer has a distinct mechanism of action that removes rather than neutralizes endogenous acid as a means of 
restoring acid-base balance in the body under the conditions of metabolic acidosis. As kidney function declines, generation of metabolic acids continues, but their excretion is compromised, leading to acid retention. Current treatment options to attenuate this acid retention include dietary interventions with very low-protein diets (Garneata et al., 2016), baseproducing fruits and vegetables (Goraya et al., 2013, 2014, 2019), or oral alkali supplementation (de Brito-Ashurst et al., 2009; Phisitkul et al., 2010; Di Iorio et al., 2019; Dubey et al., 2020). Although dietary interventions are effective in elevating serum bicarbonate and reducing kidney function decline, adherence to dietary interventions can be difficult to achieve (Garneata et al., 2016) and necessarily involves the consumption of foods high in dietary potassium (Goraya and Wesson, 2019). Oral alkali supplementation introduces a significant amount of sodium into the body, along with the neutralizing bicarbonate or citrate equivalents, increasing overall sodium load in patients with CKD. Indeed, to raise serum bicarbonate by $3-4 \mathrm{mEq} / \mathrm{l}$ in an $80-\mathrm{kg}$ patient, $6-8 \mathrm{~g} /$ day of sodium bicarbonate is required, delivering $1.7-2.2 \mathrm{~g} /$ day of sodium in addition to normal dietary sodium intake (Abramowitz et al., 2013), thus exceeding the published guidelines for daily sodium intake in patients with CKD (Wright and Cavanaugh,
2010). This additional sodium has the potential to aggravate sodium-sensitive comorbidities, such as hypertension, congestive heart failure, and edema, in patients with CKD (Bushinsky, 2019; Navaneethan et al., 2019). The additional sodium load from sodium-based oral alkali may also diminish the effect of renoprotective agents, such as renin-angiotensinaldosterone system inhibitors (Lambers Heerspink et al., 2012; Vegter et al., 2012), and lead to worsening albuminuria (Raphael et al., 2020), thereby counteracting the benefits of treating metabolic acidosis.

To avoid these challenges, veverimer removes acid through the GI tract, thus reducing the overall acid accumulation that is the hallmark of chronic metabolic acidosis, and in so doing introduces bicarbonate into the circulation without the addition of deleterious counterions. This mechanism of acid removal has similarities to the physiologic response to prolonged vomiting, chloride-losing diarrhea, or nasogastric suction, each of which removes $\mathrm{HCl}$ from the stomach and leads to a rapid increase in serum bicarbonate levels that may result in hypochloremic metabolic alkalosis (Kassirer and Schwartz, 1966; Niv and Fraser, 2002; Khanna and Kurtzman, 2006; Valeur and Julsrud, 2013). It is fundamentally different from these responses in persons with normal kidney function,

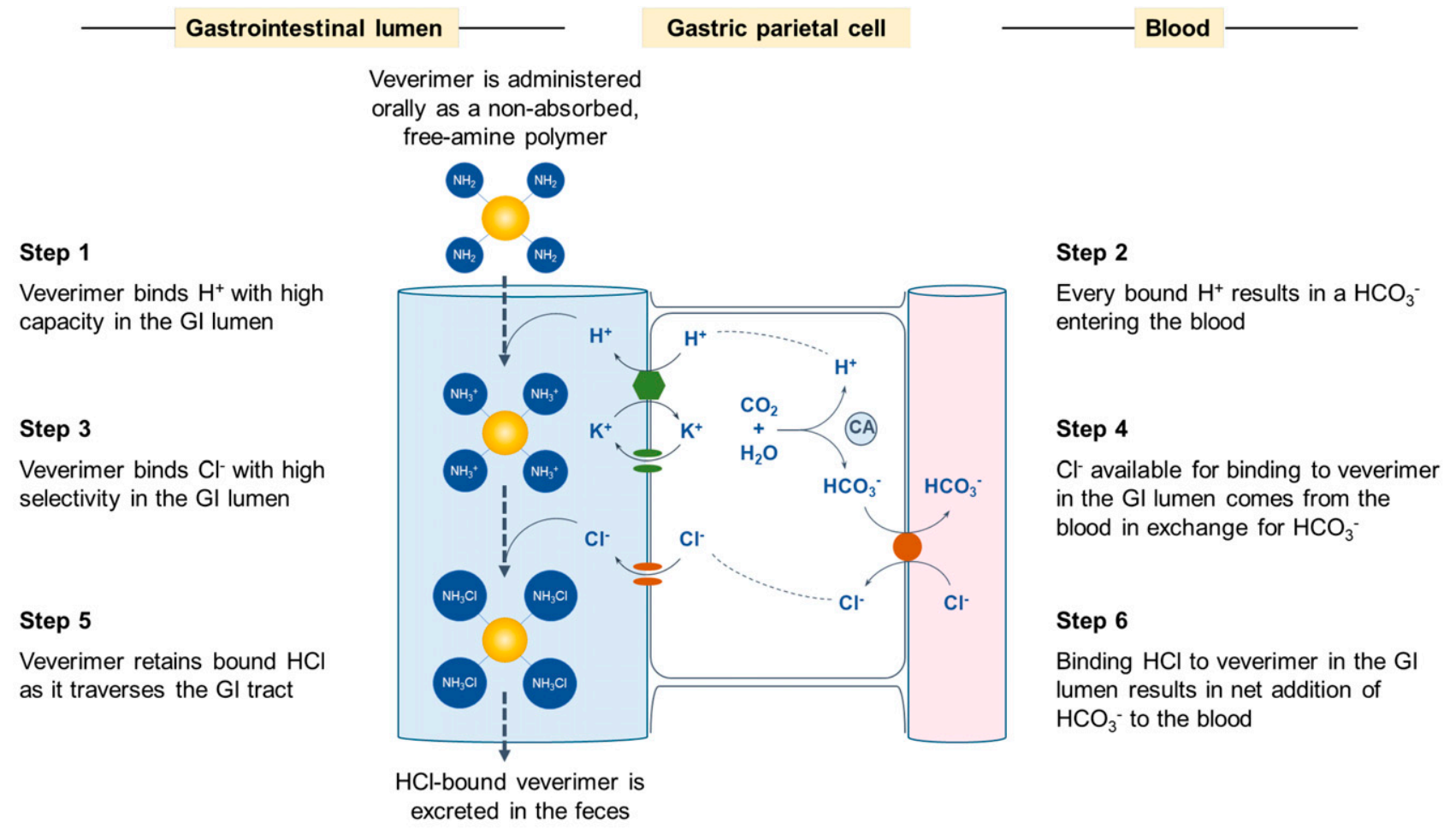

Carbonic anhydrase

$\mathrm{HCO}_{3}-\mathrm{Cl}^{-}$exchanger

$\mathrm{Cl}^{-}$channel

$\mathrm{H}^{+} / \mathrm{K}^{+}$ATPase

$\mathrm{K}^{+}$channel

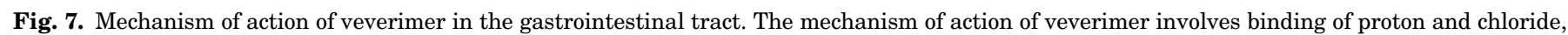

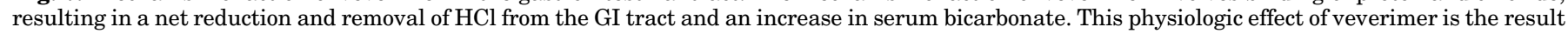

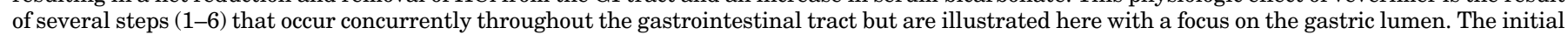

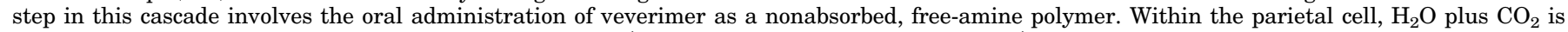

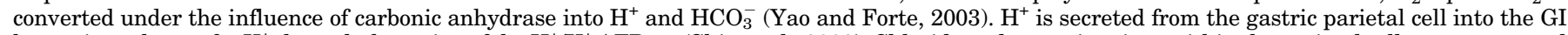

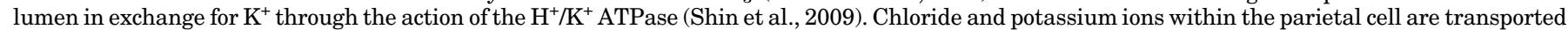

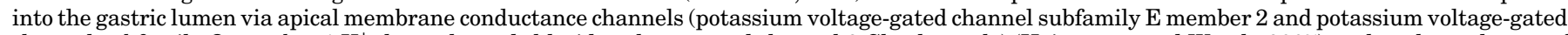

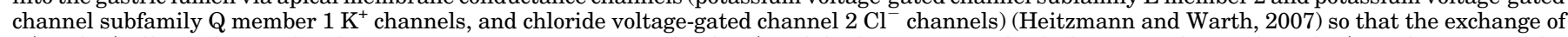

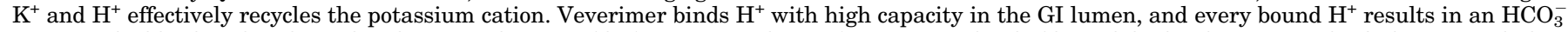

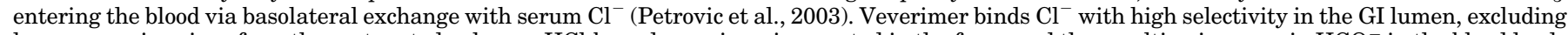

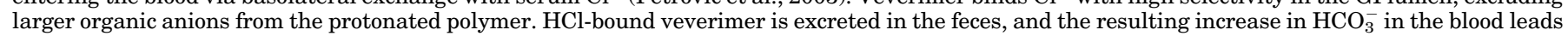
to a correction of metabolic acidosis. 
however, in that veverimer only binds $\mathrm{HCl}$ and does not induce the loss of fluid, sodium, and potassium that are hallmarks of hypochloremic metabolic alkalosis (Khanna and Kurtzman, 2006).

The selective binding and removal of $\mathrm{HCl}$ by veverimer allows for the excretion of acid while generating bicarbonate in the blood by the mechanisms described in the process of parietal cell $\mathrm{HCl}$ secretion (summarized in Fig. 7). In the process of facilitating the excretion of $\mathrm{HCl}$ through the gut in this manner, veverimer can be seen as supplementing and replacing the deficiency of acid excretion in the diseased kidney, thus extending the known structural and functional aspects of the gut epithelium that often mimic epithelial cells of the kidney nephrons (Coudrier et al., 1988; Schultheis et al., 1998; Wang et al., 2002; Alper and Sharma, 2013; Soleimani, 2013).

In conclusion, the studies reported here demonstrate that the chemical structure and design of veverimer are translated into a potent and selective binding of $\mathrm{HCl}$, as measured with in vitro matrices mimicking various aspects of the GI tract and in animal studies that measure chloride removal through the feces with a polymer that is nonabsorbed and stable throughout GI transit. The physiologic consequence of specific $\mathrm{HCl}$ removal is the elevation of serum bicarbonate. The polymer does not introduce counterions nor does it remove organic anions in the GI tract that provide sources of bicarbonate precursors once absorbed. Veverimer treatment in an animal model of $\mathrm{CKD}$ with metabolic acidosis demonstrated that $\mathrm{HCl}$ binding and removal by the polymer increased serum bicarbonate, which was consistent with completed studies in human subjects that demonstrate the potential for veverimer to treat chronic metabolic acidosis in patients with CKD.

\section{Acknowledgments}

The authors would like to acknowledge the helpful discussions, editing, and insightful comments provided by David Bushinsky (University of Rochester), Dawn Parsell (Tricida, Inc.), and Christopher Waldapfel (Tricida, Inc.) in the course of the work and in the preparation of this report.

\section{Authorship Contributions}

Participated in research design: Klaerner, Shao, Biyani, Kierstead, Tabakman, Nguyen, Buysse.

Conducted experiments: Shao, Biyani, Kade, Kierstead, Gbur, Tabakman, Nguyen.

Contributed new reagents or analytic tools: Biyani, Kade, Kierstead, Gbur, Tabakman, Nguyen.

Performed data analysis: Klaerner, Shao, Biyani, Kade, Kierstead,

Gbur, Tabakman, Nguyen, Buysse.

Wrote or contributed to the writing of the manuscript: Klaerner, Shao, Biyani, Kade, Kierstead, Gbur, Tabakman, Nguyen, Buysse.

\section{References}

Abramowitz MK, Melamed ML, Bauer C, Raff AC, and Hostetter TH (2013) Effects of oral sodium bicarbonate in patients with CKD. Clin J Am Soc Nephrol 8:714-720.

Alper SL and Sharma AK (2013) The SLC26 gene family of anion transporters and channels. Mol Aspects Med 34:494-515.

Alpern RJ and Sakhaee K (1997) The clinical spectrum of chronic metabolic acidosis: homeostatic mechanisms produce significant morbidity. Am J Kidney Dis 29 291-302.

Bushinsky DA (2019) Tolerance to sodium in patients with CKD-induced metabolic acidosis: does the accompanying anion matter? Am J Kidney Dis 73:858-865.

Bushinsky DA, Hostetter T, Klaerner G, Stasiv Y, Lockey C, McNulty S, Lee A, Parsell D, Mathur V, Li E, et al. (2018) Randomized, controlled trial of TRC101 to increase serum bicarbonate in patients with CKD. Clin J Am Soc Nephrol 13: 26-35.

Coudrier E, Kerjaschki D, and Louvard D (1988) Cytoskeleton organization and submembranous interactions in intestinal and renal brush borders. Kidney Int $\mathbf{3 4}$ 309-320. de Brito-Ashurst I, Varagunam M, Raftery MJ, and Yaqoob MM (2009) Bicarbonate supplementation slows progression of CKD and improves nutritional status. $J \mathrm{Am}$ Soc Nephrol 20:2075-2084

den Besten G, van Eunen K, Groen AK, Venema K, Reijngoud DJ, and Bakker BM (2013) The role of short-chain fatty acids in the interplay between diet, gut microbiota, and host energy metabolism. J Lipid Res 54:2325-2340.

Di Iorio BR, Bellasi A, Raphael KL, Santoro D, Aucella F, Garofano L, Ceccarelli M, Di Lullo L, Capolongo G, Di Iorio M, et al.; UBI Study Group (2019) Treatment of metabolic acidosis with sodium bicarbonate delays progression of chronic kidney disease: the UBI Study. $J$ Nephrol 32:989-1001.

Diwan V, Brown L, and Gobe GC (2018) Adenine-induced chronic kidney disease in rats. Nephrology (Carlton) 23:5-11.

Dubey AK, Sahoo J, Vairappan B, Haridasan S, Parameswaran S, and Priyamvada PS (2020) Correction of metabolic acidosis improves muscle mass and renal function in chronic kidney disease stages 3 and 4: a randomized controlled trial. Nephrol Dial Transplant 35:121-129.

Garneata L, Stancu A, Dragomir D, Stefan G, and Mircescu G (2016) Ketoanaloguesupplemented vegetarian very low-protein diet and CKD progression. J Am Soc Nephrol 27:2164-2176.

Gennari FJ and Weise WJ (2008) Acid-base disturbances in gastrointestinal disease. Clin J Am Soc Nephrol 3:1861-1868.

Goraya N, Munoz-Maldonado Y, Simoni J, and Wesson DE (2019) Fruit and vegetable treatment of chronic kidney disease-related metabolic acidosis reduces cardiovascular risk better than sodium bicarbonate. Am J Nephrol 49: $438-448$.

Goraya N, Simoni J, Jo CH, and Wesson DE (2013) A comparison of treating metabolic acidosis in CKD stage 4 hypertensive kidney disease with fruits and vegetables or sodium bicarbonate. Clin J Am Soc Nephrol 8:371-381.

Goraya N, Simoni J, Jo CH, and Wesson DE (2014) Treatment of metabolic acidosis in patients with stage 3 chronic kidney disease with fruits and vegetables or oral bicarbonate reduces urine angiotensinogen and preserves glomerular filtration rate. Kidney Int 86:1031-1038.

Goraya N and Wesson DE (2017) Management of the metabolic acidosis of chronic kidney disease. Adv Chronic Kidney Dis 24:298-304.

Goraya N and Wesson DE (2019) Clinical evidence that treatment of metabolic acidosis slows the progression of chronic kidney disease. Curr Opin Nephrol Hypertens 28:267-277.

Hamm LL, Nakhoul N, and Hering-Smith KS (2015) Acid-base homeostasis. Clin $J$ Am Soc Nephrol 10:2232-2242.

Heitzmann D and Warth R (2007) No potassium, no acid: K+ channels and gastric acid secretion. Physiology (Bethesda) 22:335-341.

Jung T, Kamm W, Breitenbach A, Kaiserling E, Xiao JX, and Kissel T (2000) Biodegradable nanoparticles for oral delivery of peptides: is there a role for polymers to affect mucosal uptake? Eur J Pharm Biopharm 50:147-160.

Kassirer JP and Schwartz WB (1966) The response of normal man to selective depletion of hydrochloric acid. Factors in the genesis of persistent gastric alkalosis. Am J Med 40:10-18.

Khanna A and Kurtzman NA (2006) Metabolic alkalosis. J Nephrol 19 (Suppl 9): S86-S96.

Kraut JA and Madias NE (2016) Metabolic acidosis of CKD: an update. Am J Kidney Dis 67:307-317.

Kraut JA and Madias NE (2018) Re-evaluation of the normal range of serum total $\mathrm{CO}_{2}$ concentration. Clin J Am Soc Nephrol 13:343-347.

Lambers Heerspink HJ, Holtkamp FA, Parving HH, Navis GJ, Lewis JB, Ritz E, de Graeff PA, and de Zeeuw D (2012) Moderation of dietary sodium potentiates the renal and cardiovascular protective effects of angiotensin receptor blockers. Kidney Int 82:330-337.

Lin SH, Cheema-Dhadli S, Chayaraks S, Chen CB, Gowrishankar M, and Halperin ML (1998) Physiological disposal of the potential alkali load in diet of the rat: steps to achieve acid-base balance. Am J Physiol 274:F1037-F1044.

Navaneethan SD, Shao J, Buysse J, and Bushinsky DA (2019) Effects of treatment of metabolic acidosis in CKD: a systematic review and meta-analysis. Clin J Am Soc Nephrol 14:1011-1020.

Niv Y and Fraser GM (2002) The alkaline tide phenomenon. J Clin Gastroenterol 35: $5-8$.

Petrovic S, Ju X, Barone S, Seidler U, Alper SL, Lohi H, Kere J, and Soleimani M (2003) Identification of a basolateral Cl-/HCO3- exchanger specific to gastric parietal cells. Am J Physiol Gastrointest Liver Physiol 284:G1093-G1103.

Phisitkul S, Khanna A, Simoni J, Broglio K, Sheather S, Rajab MH, and Wesson DE (2010) Amelioration of metabolic acidosis in patients with low GFR reduced kidney endothelin production and kidney injury, and better preserved GFR. Kidney Int 77: $617-623$.

Raphael KL (2018) Metabolic acidosis and subclinical metabolic acidosis in CKD. J Am Soc Nephrol 29:376-382.

Raphael KL, Isakova T, Ix JH, Raj DS, Wolf M, Fried LF, Gassman JJ, Kendrick C, Larive B, Flessner MF, et al. (2020) A randomized trial comparing the safety, adherence, and pharmacodynamics profiles of two doses of sodium bicarbonate in CKD: the BASE pilot trial. J Am Soc Nephrol 31:161-174.

Ríos-Covián D, Ruas-Madiedo P, Margolles A, Gueimonde M, de Los Reyes-Gavilán CG, and Salazar N (2016) Intestinal short chain fatty acids and their link with diet and human health. Front Microbiol 7:185.

Schultheis PJ, Clarke LL, Meneton P, Miller ML, Soleimani M, Gawenis LR, Riddle TM, Duffy JJ, Doetschman T, Wang T, et al. (1998) Renal and intestinal absorptive defects in mice lacking the NHE3 $\mathrm{Na}+\mathrm{H}+$ exchanger. Nat Genet 19: $282-285$

Scialla JJ and Anderson CA (2013) Dietary acid load: a novel nutritional target in chronic kidney disease? Adv Chronic Kidney Dis 20:141-149.

Shao J, Parsell D, Guttendorf R, Wu YS, Tsao L, Tabakman S, Stasiv Y, Lee A, Biyani K, and Klaerner G (2020) P0009 Evaluation of the potential for drug interactions with veverimer. Nephrol Dial Transplant 35 (Suppl 3):2. 
Shin JM, Munson K, Vagin O, and Sachs G (2009) The gastric HK-ATPase: structure, function, and inhibition. Pflugers Arch 457:609-622.

Soleimani M (2013) SLC26 Cl-/HCO3- exchangers in the kidney: roles in health and disease. Kidney Int 84:657-666.

Szerlip HM (2005) Metabolic acidosis, in National Kidney Foundation's Primer on Kidney Diseases (Gilbert SJ and Weiner DE eds) pp 130-143, Elsevier Inc., Philadelphia.

Toto RD and Alpern RJ (1996) Metabolic acid-base disorders, in Fluids and Electrolytes (Kokko JP and Tannen RL eds) pp 201-266, Saunders, Philadelphia.

Turnberg LA, Bieberdorf FA, Morawski SG, and Fordtran JS (1970) Interrelationships of chloride, bicarbonate, sodium, and hydrogen transport in the human ileum. J Clin Invest 49:557-567.

Valeur J and Julsrud J (2013) Vomiting: a physiological response to acidosis? Scand $J$ Gastroenterol 48:1103-1104.

Vegter S, Perna A, Postma MJ, Navis G, Remuzzi G, and Ruggenenti P (2012) Sodium intake, ACE inhibition, and progression to ESRD. J Am Soc Nephrol 23 $165-173$.

Wang Z, Petrovic S, Mann E, and Soleimani M (2002) Identification of an apical Cl()/HCO3(-) exchanger in the small intestine. Am J Physiol Gastrointest Liver Physiol 282:G573-G579.
Wesson DE, Buysse JM, and Bushinsky DA (2020) Mechanisms of metabolic acidosis-induced kidney injury in chronic kidney disease. J Am Soc Nephrol 31:469-482.

Wesson DE, Mathur V, Tangri N, Stasiv Y, Parsell D, Li E, Klaerner G, and Bushinsky DA (2019a) Long-term safety and efficacy of veverimer in patients with metabolic acidosis in chronic kidney disease: a multicentre, randomised, blinded, placebo-controlled, 40-week extension. Lancet 394:396-406.

Wesson DE, Mathur V, Tangri N, Stasiv Y, Parsell D, Li E, Klaerner G, and Bushinsky DA (2019b) Veverimer versus placebo in patients with metabolic acidosis associated with chronic kidney disease: a multicentre, randomised, doubleblind, controlled, phase 3 trial. Lancet 393:1417-1427.

Wright JA and Cavanaugh KL (2010) Dietary sodium in chronic kidney disease: a comprehensive approach. Semin Dial 23:415-421.

Yao X and Forte JG (2003) Cell biology of acid secretion by the parietal cell. Annu Rev Physiol 65:103-131.

Address correspondence to: Jun Shao, Tricida, Inc., 7000 Shoreline Ct., Suite 201, South San Francisco, CA 94080. E-mail: jshao@tricida.com 